\title{
Different structures formed at HII boundaries
}

\author{
Jingqi Miao $\dagger$, Paul Cornwall and Tim Kinnear \\ The School of Physical Sciences, University of Kent, Canterbury, CT2 7NH, UK \\ email: J.Miao@kent.ac.uk
}

\begin{abstract}
Hydrodynamic simulations on the evolution of molecular clouds (MCs) at HII boundaries are used to show that radiation driven implosion (RDI) model can create almost all of the different morphological structures, such as a single bright-rimmed cloud (BRC), fragment structure and multiple elephant trunk (ET) structures.
\end{abstract}

Keywords. ISM: molecular cloud, HII regions, ISM: structure

The interaction of UV radiation with MCs creates a diversity of morphological structures at HII boundaries which prompted several theoretical models, such as the RDI model (Lefloch \& Lazareff 1994) for the formation of a single BRC (Sugitani \& Ogura 1994), collect \& collapse (C\&C) model (Elmegreen \& Lada 1977) for HII bubbles or fragments (Deharveng, Zavagno, \& Caplan 2005) and hydrodynamic instability model (HD) for a forest of ETs (White et al. 1999). A mixture of the above mentioned structures are often found in one HII region (Chauhan et al. 2011), so it is desirable to use one model to consistently interpret the overall structure.

Using an existing numerical (Miao et al. 2009) code based on RDI mechanism and smoothed particle hydrodynamic ( $\mathrm{SPH}$ ) method, and considering the clumpiness and non-spherical nature of a MC, we found that a prolate uniform MC could evolve into a bar-like structure with condensed cores embedded along the fragment when its major axis is perpendicular to the UV radiation field. An asymmetrical BRC with one side compressed more strongly than the other side will form, if its major axis is initially inclined to an UV radiation flux by an angle. Triggered single or multi star(s) are found embedded at the centre of the head of the BRCs. These simulation results well interpret the physical features of some of the BRCs observed. Further investigation shows that a clumpy $\mathrm{MC}$ with proper initial conditions could form various ET structures.

Our simulation results, combined with that of Walch et al. (2011) on the formation of HII bubble structure state that RDI mechanism is a versatile model to deal with almost all of the morphological structure formation at HII boundaries.

\section{References}

Chauhan, N., Ogura, K., Pandey, A. K., et al. 2011, PASJ 63, 795

Deharveng, L., Zavagno, A., \& Caplan, J. 2005, A\&A 433, 565

Elmegreen, B. G. \& Lada, C. J. 1977, ApJ 214, 725

Lefloch, B. \& Lazareff, B. 1994, A\&\&A 289, 559

Miao, J., White, G. J., Thompson, M. A., \& Nelson, R. P. 2009, MNRAS 692, 382

Sugitani, K. \& Ogura, K. 1994, ApJS 92, 163

Walch, S., Whitworth, A., Bisbas, T., Hubber, D. A., \& Wuensch, R. 2011, 2011arXiv $1109.3478 \mathrm{~W}$

White, G. J., Nelson, R. P., Holland, W. S., et al. 1999, A\&A 343, 233

$\dagger$ Present address: The Centre for Astrophysics \& Planetary Science, CT2 7NH UK 